\title{
Synthesis of Full Order Observer for Vector Control System of Induction Motor Drive
}

\author{
Kucher Ekaterina S. \\ Department of Electric Drives and Industry Automation \\ Novosibirsk State Technical University \\ Novosibirsk, Russian Federation \\ kucher@corp.nstu.ru
}

\begin{abstract}
Paper presents method for synthesis of full order observer, which allows for parametric synthesis of observing algorithms for multi-input/multi-output (MIMO) control systems, such as the vector control system of induction motor drive. This method on the principle of motion separation and on the Akkermanh formula is based.

Under the influence of uncontrolled signal and parametric disturbances, the synthesized speed control system should ensure the proximity of processes to the desired, as well as the static accuracy of performance. Results can be obtained using special method of structural and parametrical synthesis, such as method of localization.
\end{abstract}

Keywords- induction motor drive, full order observe, Akkermanh formula, MIMO control system, slow movement loop, fast movement loop, localization method.

\section{INTRODUCTION}

At present, the wide use of induction motor drives is due to the application of the mathematical model for vector control.

This approach to the control of ac drives requires knowledge of the exact values of many parameters for the scheme of replacing induction motors. Besides, for the realization of vector control system, it is necessary to calculate the vector of the main flux linkage or rotor flux linkage.

Today, there is a trend to exclude the sensors of the coordinates of the mechanical and magnetic state of electric drives from the structure of vector control systems by attracting of algorithms for observation and identification [1].

Speed estimation is an issue of particular interest with induction motor drives where the mechanical speed of the rotor is generally different from the speed of the revolving magnetic field. The advantages of speed sensorless induction motor drives are reduced hardware complexity and lower cost, the reduced size of the drive machine, elimination of the sensor cable, better noise immunity, increased reliability and less maintenance requirements. The operation in hostile environments mostly requires a motor without a speed sensor.

The processes of observing the reference vector of flux linkage and the current identification of the parameters of the induction motor drives are become more complicated in the sensorless electric drives that do not have sensors of the mechanical position of the rotor or the motor speed.

Most methods of sensorless vector control are based on the indirect determination of the electric position of the rotor of an induction motor. To calculate the vector of flux linkage or the position of the rotor for induction motor drive, in practice, the most commonly used are adaptive observers of the state of full and reduced order (Full Order and Reduce State Observer).

Great attention is paid to construction of control systems, taking into account the importance of factors of uncertainty, which include inconsistencies in the aprioristic information on values of parameters for the model, influence of external indignations. Also it is necessary to consider incompleteness of the information by a current condition of the object.

The greatest efficiency of stabilization of control of nonstationary objects is provided with the techniques based on a principle of deep feedback which allow setting not only static accuracy, but also desirable indicators of quality for transients of the control system. In such methods, it will be purposeful to organize slow and rapid movements of processes by means of which, after carrying out certain transformations, it is possible to isolate them into separate contours.

The contour of fast movements localizes both external and parametrical indignations -properties of object, and is the most internal contour of the control system.

Slow movements fully meet the requirements, and synthesis of the contours carried out implicitly when choosing parameters of the equation of desirable movements. This contour provides for a small sensitivity to changes in the parameters of the object.

When constructing a control system by using the principle of separation of motion, it is possible to decompose the synthesis problems of the contours considered above, thereby simplifying the analysis of the solution - the contours of slow and fast movements can be synthesized separately from each other.

As a two-channel non-stationary object, the model of an asynchronous motor in a fixed coordinate system will be considered. It gives the chance to operate independently target variables of the object, i.e. a magnetic condition and the electromagnetic moment of the induction motor drive. 
This paper presents a structural and parametrical synthesisc full order state observer method by the extended Ackermann formula for the pole-placement of a MIMO system is presented in this paper. The proposed method can determine desired feedback gain matrices without utilizing any similarity transformation of control system of induction motor drives using the localization method. It also analyzes the results of studies of organized control system, obtained with the help of digital simulation.

\section{MATHEMATICAL DESCRIPTION OF OBJECT}

The use of spatial vectors as complex state variables is an efficient method for ac machine modeling. Vector controlled drives require estimating the magnitude and spatial orientation of the fundamental magnetic flux waves in the stator or in the rotor. They differ with respect to accuracy, robustness, and sensitivity to variations in model parameters. Dynamic performance and steady-state speed accuracy in the low speed range can be achieved by exploiting parasitic effects of the machine [2].

The principle of space vector control is based on the mathematical model of the $\mathrm{AC}$ motor which allows presenting the engine as a two-channel object of control in the field oriented orthogonally the system of coordinates on a vector of the flux linkage. It gives the chance to operate independently variables of the object, i.e. a magnetic condition and the electromagnetic moment of the AC machine.

Let us consider the mathematical description of the ac engine in the field oriented system of coordinates $(d, q)$ rotated relatively motionless coordinate system $(\alpha, \beta)$ at such angle $\gamma_{\psi}$ at which longitudinal axis $d$ coincides in the direction with the vector of flux linkage $\Psi_{r}$. When using system $(d, q)$, the cyclic speed of rotating system coordinates is equal to instant speed of a vector of flux linkage rotor $\omega_{k}=\omega_{\psi}$, and the equations of the induction motor (1) in the scalar form of the recording take the form $[1,2,3,4]$ :

$$
\left.\begin{array}{c}
L_{\sigma e} \cdot \frac{d i_{s d}}{d t}=-R_{s} \cdot i_{s d}-\frac{L_{m}}{L_{r}} \cdot \frac{d \psi_{r m}}{d t}+\omega_{\psi} \cdot L_{\sigma e} \cdot i_{s q}+u_{s d} \\
L_{\sigma e} \cdot \frac{d i_{s q}}{d t}=-R_{s} \cdot i_{s q}-\omega_{\psi} \cdot\left(L_{\sigma e} \cdot i_{s d}+\frac{L_{m}}{L_{r}} \cdot \psi_{r m}\right)+u_{s q} \\
\frac{d \psi_{r m}}{d t}=\frac{L_{m}}{T_{r}} \cdot i_{s d}-\frac{1}{T_{r}} \cdot \psi_{r m} \\
\frac{d \gamma_{\psi}}{d t}=\omega_{\psi} \\
M_{e}=p_{n} \cdot \frac{L_{m}}{L_{r}} \cdot \psi_{r m} \cdot i_{s q}
\end{array}\right\}
$$

where $\psi_{r m}, \omega_{\psi}$ - Euclidean norm and instantaneous circular frequency of the rotor flux linkage vector; $i_{s d}, i_{s q}-$ magnetizing and torque-forming components of the stator currents vector; $u_{s d}, u_{s q}$ - components of the stator voltage vector; $\omega_{s}-$ rotor slip frequency; $\omega_{k}$ - frequency of $\mathrm{k}$ - coordinates; $\omega_{e}$ - electric rotor speed; $D$ - rotation matrix; $L_{r}, L_{m} \cdot R_{r}, R_{s}, L_{\sigma e}-$ parameters of the AC engine ; $T_{r}=\frac{L_{r}}{R_{r}}$ - rotor time constant of the induction machine, $L_{\sigma e}-$ equivalent motor leakage inductance.

For completeness of the mathematical description of the $\mathrm{AC}$ machine, it is also necessary to consider the equation of movement of the electric motor drive:

$$
J \cdot \dot{\omega}=M_{e}-M_{c}
$$

where $M_{e}$ - electromagnetic torque, $J$ - total moment of inertia; $M_{c}$ - load torque, $p_{n}$ - number of pole pairs.

The principle of vector space control allows one to influence independently $i_{s d}-$ magnetizing and $i_{s q}$ - torqueforming components of the stator currents vector for control magnetic condition of the AC machine and the electromagnetic moment accordingly, as in the direct current engine.

\section{STRUCTURAL SYNTHESIS OF SPEED CONTROL SYSTEM OF INDUCTION MOTOR DRIVE}

Many schemes are based on the simplified engine models. They have been devised to sense the speed of the induction motor of the measured terminal quantities for control purposes. In order to obtain an accurate dynamic representation of the motor speed, it is necessary to base the calculation in the coupled circuit equation of the motor. [3]

Synthesis of the speed control system of the induction motor drive is carried out on the basis of the principle for subordinate regulation, then the structural scheme of the system vector control will look as shown in Fig. 1.

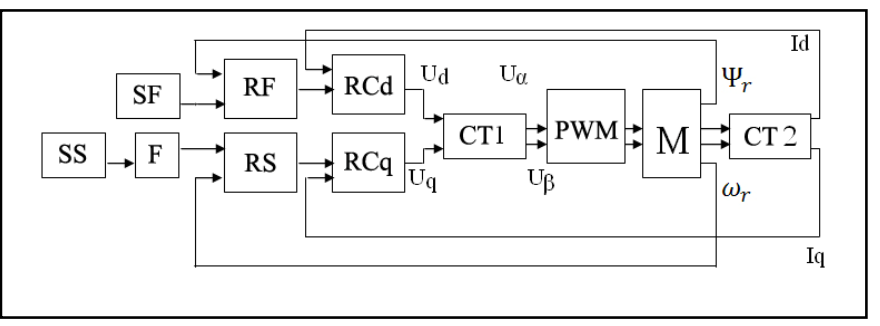

Fig. 1. Structural scheme of system vector control

Fig. 1shows RF - flux linkage regulator, $\mathrm{RCd}$ - regulator of magnetizing component stator current, $\mathrm{RCq}$ - regulator of component stator current, $\mathrm{RS}$ - speed regulator, $\mathrm{F}$ - filter, $\mathrm{M}$ $\mathrm{AC}$ machine, SF - setting signal in the flux linkage control channel, SS - setting signal in the speed control channel, CT1 - coordinate transformer, CT2 - coordinate transformer.

\section{PARAMETRIC SYNTHESIS OF THE CONTROL SYSTEM OF INDUCTION MOTOR DRIVE BY THE LOCALIZATION METHOD}

To illustrate the method idea, let us assume that the state vector and the highest derivative $x^{(n)}$ of the controlled 
coordinate are accessible to direct measurements. The control error is given by $[4,5]$ :

$$
\varepsilon=x_{g}^{(n)}-x^{(n)}
$$

where $x_{g}^{(n)}=F(v, x)$ - equation of desired motion of the highest derivative controlled coordinate; $v(t)-$ setting influence.

The stator current control loop with a proportional-integral regulator on the longitudinal and cross axes describes the following system of equations $[4,5,7]$ :

$$
\left\{\begin{array}{c}
i_{s q}^{(1)}=K_{P} \mathcal{E}^{(1)}+K_{I} \varepsilon \\
i_{s q}^{(2)}+A_{1} \Omega_{g} i_{s q}^{(1)}+\Omega_{g}^{2} i_{s q}=\Omega_{g}^{2} U_{S C} \\
T_{f}^{2} \hat{i}_{s q}^{(2)}+A_{1 f} T_{f} \hat{i}_{s q}^{(1)}+\hat{i}_{s q}=i_{s q}
\end{array}\right.
$$

The first equation of system (3) is the control law, where $K_{P}, K_{I^{-}}$coefficients of the proportional and integral regulator part. The following equation is the system equations of desired motions, where $A_{1}$ - coefficient of desired motion; $\Omega_{g}$ - compound root of desired motion; $U_{S C}-$ setting influence of stator current. The third equation of system (3) is the mathematical description of the differentiating filter.

Let us obtain the equation of stator current error from the equation of desired motion:

$$
\begin{gathered}
i_{s q}^{(2)}=\Omega_{g}^{2} U_{S C}-A_{1} \Omega_{g} i_{s q}^{(1)}-\Omega_{g}^{2} i_{s q}, \\
\varepsilon=i_{s q}^{(2)}-\hat{i}_{s q}^{(2)} .
\end{gathered}
$$

To find the coefficients of the proportional and integral regulator part, let us make the structural synthesis of the stator current control loop in accordance with the system of equations (3) and (4) (Fig. 2).

Let us accept that $\frac{K_{n}}{\tau_{\mu} p+1} \approx K_{n}, D_{f}(p) \approx T_{f} p+1$.

Then the characteristic equation of fast movement of the inner contour (Fig. 2) is equated to the normalized characteristic equation of the fast motions subsystem, which has the following form [5]:

$$
T_{b}^{3} p^{3}+T_{b}^{2} A_{2 b} p^{2}+T_{b} A_{1 b} p+1=0,
$$

where $T_{b} \leq \frac{T_{g}}{\eta}-$ time constant of the fast movement loop, $T_{g}=\frac{1}{\Omega_{g}}-$ time constant of desired motions; $A_{i b}-$ characteristic polynomial coefficient.

Taking into account the value of the time differentiation filter, the degree for separation of motion and the coefficients of the characteristic polynomial fast movement loop is calculated as the coefficients of the proportional and integral part of the current regulator:

$$
K_{I}=\frac{R_{s} T_{f} T_{\sigma e} \Omega_{g}^{3} \eta^{3}}{K_{n}}, K_{P}=\frac{T_{b} A_{1 b} K_{I} K_{n}-R_{s}}{K_{n}}
$$

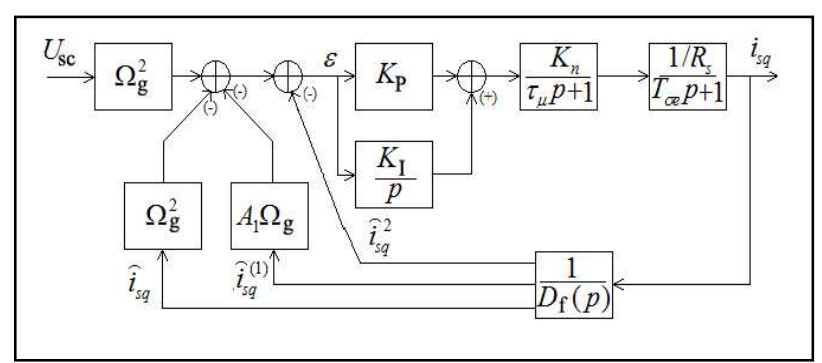

Fig. 2. Structural scheme of the stator current control loop

The regulation loop of the rotor flux-linkage with the proportional-integral regulator is also described by three equations:

$$
\left\{\begin{array}{c}
U_{s d}^{(1)}=K_{P} \varepsilon^{(1)}+K_{I} \varepsilon \\
\psi_{r}^{(2)}+A_{1} \Omega_{g} \psi_{r}^{(1)}+\Omega_{g}^{2} \psi_{r}=\Omega_{g}^{2} U_{S F} \\
T_{f}^{2} \widehat{\psi}_{r}^{2}+A_{1 f} T_{f} \widehat{\psi}_{r}^{(1)}+\widehat{\psi}_{r}=\psi_{r}
\end{array}\right.
$$

where $U_{S F}$ - setting influence of the rotor flux linkage; $\widehat{\psi}_{r}-$ estimation of the rotor flux linkage.

As well as in the stator current loop, the equation for the error in regulation of the rotor flux linkage is obtained from the equation of desired motions:

$$
\varepsilon=F(v, x)-\widehat{\psi}_{r}^{(2)}
$$

Using the system of equations (5) and (7), let us construct the structural scheme of the control loop of rotor flux and find the coefficients of the flux proportional-integral regulator (Fig. 3).

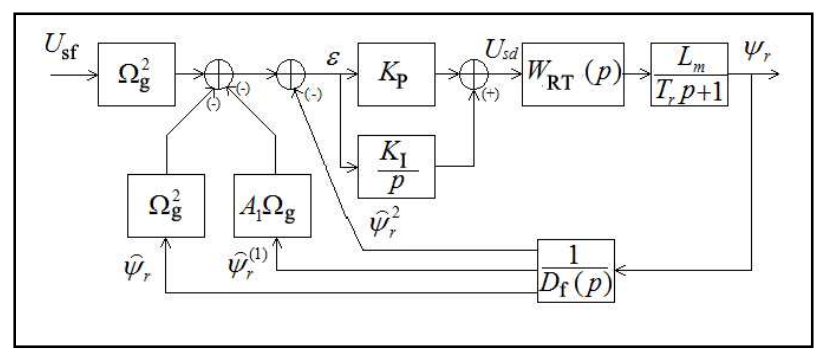

Fig. 3. Structural scheme of the rotor flux control loop

Further, according to the method described above, the characteristic polynomial of the inner contour of movements (Fig. 3) is equated to the normalized polynomial of the form (5) and the coefficients of the proportional-integral regulator of the flux linkage is calculated, while it is necessary to set the filter time constant, the degree for separation of motion and the normalized polynomial coefficients of the fast movement loop: 


$$
K_{I}=\frac{T_{r} T_{f} K_{o c t} \Omega_{g}{ }^{3} \eta^{3}}{L_{m}}, K_{P}=\frac{T_{b} A_{1 b} K_{I} L_{m}-K_{o c t}}{L_{m}} .
$$

When synthesizing the rotor speed control loop, it is necessary to specify the same three equations:

$$
\left\{\begin{array}{c}
U_{s q}^{(1)}=K_{P} \varepsilon^{(1)}+K_{I} \varepsilon \\
\omega_{r}^{(2)}+A_{1} \Omega_{g} \omega_{r}^{(1)}+\Omega_{g}^{2} \omega_{r}=\Omega_{g}^{2} U_{S C} \\
T_{f}^{2} \widehat{\omega}_{r}^{2}+A_{1 f} T_{f} \widehat{\omega}_{r}^{(1)}+\widehat{\omega}_{r}=\omega_{r}
\end{array}\right.
$$

where $U_{S C}-$ setting influence of the speed rotor; $\widehat{\omega}_{r}-$ estimation of the speed rotor.

The expression for determining the speed control error will have the form:

$$
\varepsilon=F(v, x)-\widehat{\omega}_{r}^{(2)} .
$$

Using the system of equation (8) and expression (9), let us make structural synthesis of the rotor speed control loop of the induction motor drive (Fig. 4).

Parametric synthesis of the contour under consideration is carried out according to the method described above, which was applicable for determining parameters of the stator current control loops and the rotor flux linkage coupling of the asynchronous motor.

Expressions determine the speed regulator coefficients of the proportional and integral part:

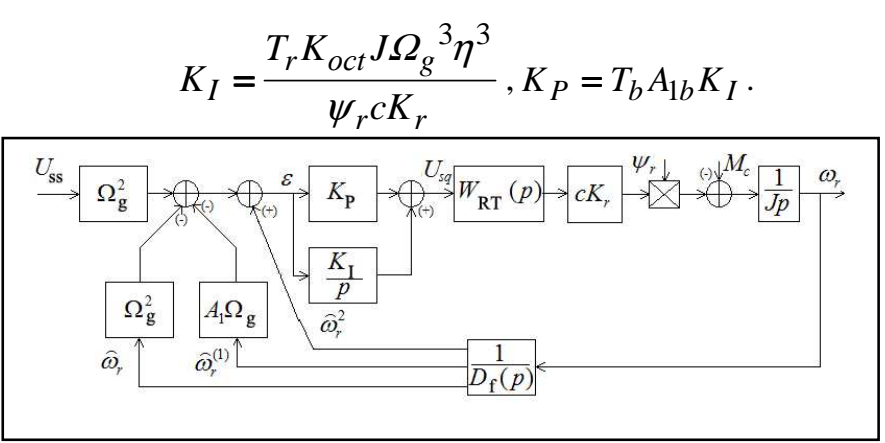

Fig. 4. Structural scheme of rotor speed control loop

\section{MATHEMATICAL DESCRIPTION OF INDUCTION MOTOR DRIVE}

The use of full order algorithms will make it possible to calculate the estimates of the reference flux vector and the rotor speed of the electric drive.

The basis of the observation processes for estimating the coordinates of the motor state are electrical quantities such as currents and stator voltages that are expose to direct measurement in the internal structure and at the output terminals of the controlled frequency converter of electrical energy.

As the control object, the induction motor model is considered, which is represented, in accordance with the principles of vector control, as a two-channel control object.
The mathematical description of the model of an induction motor drive in a fixed coordinate system will have the following form:

$$
\left.\begin{array}{l}
L_{\sigma e} \cdot \dot{I}_{s}=-R_{s} \cdot I_{s}-\frac{L_{m}}{L_{r}} \cdot \dot{\Psi}_{r}- \\
-\omega_{k} \cdot D \cdot\left(L_{\sigma e} \cdot I_{s}+\frac{L_{m}}{L_{r}} \cdot \Psi_{r}\right)+U_{s}, \\
\dot{\Psi}_{r}=\frac{L_{m} \cdot R_{r}}{L_{r}} \cdot I_{s}-\left(\omega_{k}-\omega_{e}\right) \cdot D \cdot \\
-\Psi_{r}-\frac{R_{r}}{L_{r}} \cdot \Psi_{r} .
\end{array}\right\}
$$

where $i_{s \alpha}, i_{s \beta}$ - magnetizing and torque-forming components of the stator currents vector in the accepted coordinate system; $u_{s \alpha}, u_{s \beta}$ - components of the stator voltages vector; $\omega_{k}-$ angular speed of the coordinate system; $\omega_{e}$ - electric rotor speed; $D$ - rotation matrix; $L_{r}, L_{m} \cdot R_{r}, R_{s}, L_{\sigma e}$-induction motor parameters; $L_{\sigma e}$ - equivalent motor leakage inductance.

\section{Vector-Matrix Mathematical Description of INDUCTION MOTOR DRIVE}

If a system is state observable, then the poles of the closedloop state observer can be placed at any desired locations by means of observer feedback through an appropriate observer feedback gain matrix. Block transformation matrices for transforming a class of multi-input/multi-output (MIMO) state equations in general coordinates to the block controller companion form and the block observer companion form so that the pole-placement design concept of a MIMO system can be performed.

Some similarity transformation matrices discussed by Luenberger are required for determining the desired feedback gain matrices for a lass of MIMO systems. Transforming a general class of MIMO systems to the Luenberger observer canonical form for determining the state (observer) feedback gain matrices which are not unique for pole-placement. However, the Luenberger transformation method involves the products of high-dimensional system matrices which could potentially have a large condition number. Hence, the direct use of the Luenberger's method would introduce large computational errors and should be avoided.

To overcome the above drawbacks, parametrical synthesis method by the extended Ackermann formula for the poleplacement of a MIMO system is presented in this paper. The proposed method can determine desired feedback gain matrices without utilizing any similarity transformation $[6,7]$.

The presented mathematical model of the AC motor in the vector-matrix form notation will have the form:

$$
\left\{\begin{array}{c}
\dot{X}=A X+B U \\
Y=C X .
\end{array}\right.
$$


where $X=\left[\begin{array}{c}I_{s} \\ \Psi_{r}\end{array}\right]-$ vector-column of the control object state coordinates; $A=\left[\begin{array}{ll}A_{11} & A_{12} \\ A_{21} & A_{22}\end{array}\right]$ - the control object matrix, $\operatorname{dim} A=n \times n, n=4-$ the control object order; $k-$ the number of output variables; $B-$ control matrix, $\operatorname{dim} B=n \times k ; C_{-}$output matrix, $\operatorname{dim} C=k \times n$, $C=\left[\begin{array}{llll}1 & 0 & 0 & 0 \\ 0 & 1 & 0 & 0\end{array}\right] ; Y-$ vector of output measured variables; $E_{2}=\left[\begin{array}{ll}1 & 0 \\ 0 & 1\end{array}\right]$ - identity matrix; $U-$ control signals vector [8]:

$$
\begin{gathered}
A_{12}=\left[\begin{array}{cc}
\frac{L_{m} R_{r}}{L_{r}^{2} L_{\sigma e}} & \omega_{e} \frac{L_{m}}{L_{r} L_{\sigma e}} \\
-\omega_{e} \frac{L_{m}}{L_{r} L_{\sigma e}} & \frac{L_{m} R_{r}}{L_{r}^{2} L_{\sigma e}}
\end{array}\right], \\
A_{11}=\left[\begin{array}{cc}
-\frac{R_{s}}{L_{\sigma e}}-\frac{L_{m}^{2} R_{r}}{L_{r}^{2} L_{\sigma e}} & 0 \\
0 & -\frac{R_{s}}{L_{\sigma e}}-\frac{L_{m}^{2} R_{r}}{L_{r}^{2} L_{\sigma e}}
\end{array}\right], \\
A_{21}=\left[\begin{array}{cc}
\frac{L_{m} R_{r}}{L_{r}} & 0 \\
0 & \frac{L_{m} R_{r}}{L_{r}}
\end{array}\right], A_{22}=\left[\begin{array}{cc}
-\frac{R_{r}}{L_{r}} & -\omega_{e} \\
-\omega_{e} & -\frac{R_{r}}{L_{r}}
\end{array}\right],
\end{gathered}
$$

\section{MATHEMATICAL DESCRIPTION OF FULL ORDER OBSERVER}

The full order observer is a dynamic subsystem that will look like:

$$
\left\{\begin{array}{c}
\dot{\hat{X}}=A \hat{X}+B U+L(Y-\hat{Y}) \\
\hat{Y}=C \hat{X}
\end{array}\right.
$$

where $\hat{Y}$ - vector of output variable estimates; $L$ - matrix of stabilizing additive coefficients, $\operatorname{dim} L=n \times k$ [8].

As the measured variables the vector-column of stator motor currents has been selected $Y=I_{s}=\left[\begin{array}{c}i_{s \alpha} \\ i_{s \beta}\end{array}\right]$.

The calculation of the estimation of vector is due to the fact that the discrepancy between the estimated and measured values can be used as a basis for calculating the rotor speed estimation or for the online identification of the parameters of the induction motor drive replacement scheme. The mismatch of the stator currents values happen as a consequence of the influence on the considered control object of both signal and parametric perturbations, the exact value of which can't be estimated.

The desired distribution of the characteristic equation roots of the full order observer is given by the elements of the matrix stabilizing additive L, provided that the control object under consideration is completely observable by the $\mathrm{R}$. Kalman theorem [8].

\section{VIII.PARAMETRICAL SYNTHESIS OF FULL ORDER OBSERVER}

Ackermann formula for a single-input single-output (SISO) system can be extended to a class of MIMO system. In this way, the extended Ackermann formula presented in this paper for a class of MIMO systems can be employed for the case of a general class of MIMO systems [7].

The implementation of a unique parametric synthesis of control and observation algorithms for MIMO objects or systems is possible using a technique that is based on the principle of motion separation and the Juergen Ackermann formula $[6,7]$

$$
L=N(A)\left[\begin{array}{c}
C \\
C A \\
\cdots \\
C A^{n-1}
\end{array}\right]^{-1}\left[\begin{array}{c}
0_{2} \\
0_{2} \\
\cdots \\
E_{2}
\end{array}\right],
$$

where $\Omega_{H}$ - average geometric root of the characteristic polynomial of the observer; $A_{n-1}, \ldots, A_{1}$ - root distribution coefficients, which provide previously known indicators of the quality of transient processes [8];

$$
N(p)=p^{n}+A_{i-1} \cdot \Omega_{H} \cdot p^{n-1}+\ldots+A_{1} \cdot \Omega_{H}{ }^{n-1} \cdot p+\Omega_{H}{ }^{n}-
$$
characteristic normalized polynomial;

$$
\begin{gathered}
N(A)=A^{n}+A_{i-1} \cdot \Omega_{H} \cdot A^{n-1}+\ldots+A_{1} \cdot \Omega_{H}{ }^{n-1} \cdot A+\Omega_{H}{ }^{n} \cdot E ; \\
E=\left[\begin{array}{ll}
E_{2} & 0_{2} \\
0_{2} & E_{2}
\end{array}\right], \operatorname{dim} A=\operatorname{dim} E=n \times n .
\end{gathered}
$$

The presented technique, as well as the Luenberger method of synthesis, makes it possible to distribute the roots of the characteristic equation in the desired way, by using the coefficients of the standard forms of root distribution.

As a result of the calculations, the matrix of the stabilizing additive coefficients of the observer of full order was obtained:

$$
L=\left[\begin{array}{cc}
l_{1} & -l_{2} \\
l_{2} & l_{1} \\
l_{3} & -l_{4} \\
l_{4} & l_{3}
\end{array}\right] .
$$

The presented technique of parametric synthesis of a full order observer allowed one to uniquely calculate the values of the stabilizing additive coefficients for a MIMO control object. And also, to provide not only the required speed, but also the desired indicators of the quality of the transient 
process of estimating the coordinates of the state of the considered induction motor model.

\section{RESEARCH RESULTS}

Approbation of the received results of structural and parametric synthesis was carried out by means of digital modeling with the help of the MATLAB Simulink program. A digital simulation of the vector control system, closed through a full order observer, synthesized using the Ackermanh formula and the principle of motion separation was produced.

The results of approbation for developed algorithms are graphs of the rotor speed transient processes and the rotor flux linkage module estimation of asynchronous motors (Fig.5 and Fig.6), which show that full order observer Structural and parametric synthesis of control systems that are insensitive to changes in the induction motor drive parameters was carried out for a general industrial induction motor drive of medium power with different rotational rotor speeds.

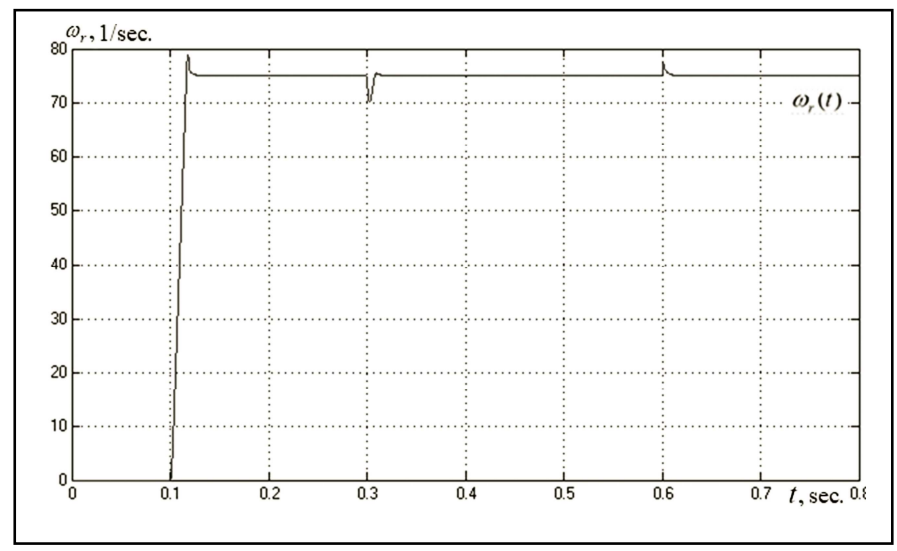

Fig. 5. Transition processes of estimation for rotor speed

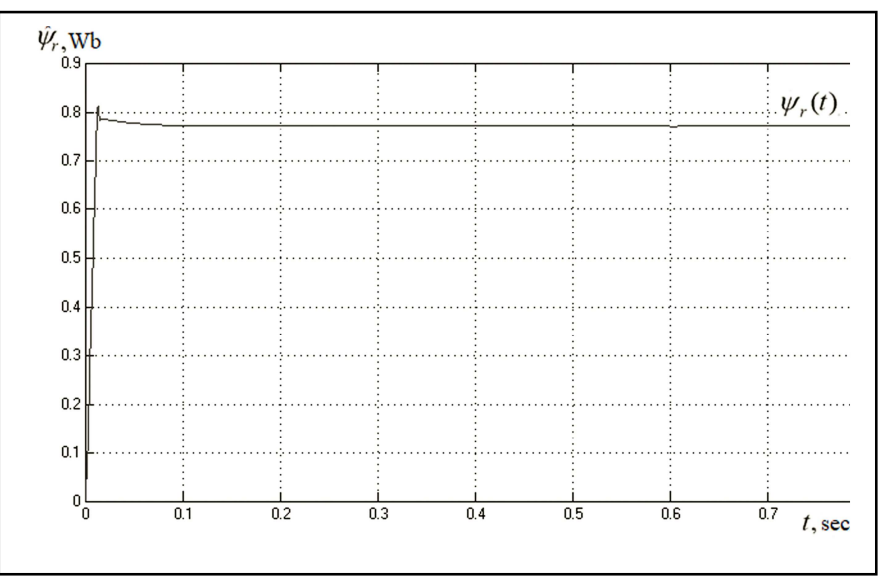

Fig. 6. Transition processes of estimation for rotor flux linkage

The calculated estimate of the rotor flux linkage vector of the induction motor allows realizing of the electric drive vector control. The received signal of the estimating the stator currents vector of the motor makes it possible to calculate in turn an estimate of the rotor rotation speed of the induction motor drive and thereby allows excluding from the structure of the vector control system the rotor position sensor of the AC motor.

\section{CONCLUSION}

Proposed methods for synthesis of control system make it possible to provide low sensitivity to introduction of parametric perturbations with the active resistance of the stator winding and the rotor winding of the induction motor drive.

The establishment of "deep" feedbacks in synthesized control systems is possible for localized effect of changes in the electric motor drive parameters by $(10-15) \%$ of nominal values. With the increase of changes in parametric disturbances, as well as the extended range of the regulation rotor speed of the induction motor drive, it is necessary to use special algorithms for parametric on-line identification and adaptation.

The proposed method of parametric synthesis of a full-order observer allows one to uniquely determine the coefficients of the stabilizing additive for the MIMO control systems.

Similar results of parametric synthesis of observation algorithms were obtained by the technique by Kubota [9].

The extended Ackermann formula is first developed for determining feedback control laws of multivariable control systems. By using the proposed methods, the desired state feedback gain matrix and the observer feedback gain matrix can be quickly determined without using any similarity transformation. The proposed method will enhance the application of pole-placement concepts to a general class of multi- variable control systems [8].

\section{References}

[1] K.Rajashekara, A.Kawamura, K.Matsuse, "Sensorless control of AC motor drives,speed and position sensorless operation, IEEE Press, pp. 1$17,1996$.

[2] J.Holtz,"Sensorless Control of induction motor drives", IEEE,vol. 90, no. 8, pp. 1358-1394, 2002.

[3] X.Xu, D.Novotny, "Implementation of direct stator flux orientation control on a versatile DSP based system", IEEE Transactions on Industry Applications, Vol. 27, No4,pp. 694-700, 1991.

[4] V.V. Pankratov, Y.A. Zima,"Multi-objective optimization of fieldoriented control systems for induction motor electric drives", Proceedings of 1st Russian - Korean International Symposium on Applied Mechanics RUSKO-AM-2001, pp. 157 - 160, October 2001.

[5] Y.A. Zima, V.V. Pankratov,"Synthesis of astatic multivariable systems by the localization method", Actual Problems of Electronics Instrument Engineering, Vol. 1, pp. 236 - 240, 2002.

[6] J.E. Ackermann, "Pole placement control", Control System, Robotics and Automation, Vol. 8, pp 74-101, 2011.

[7] H-K. Chiang et al., "Extended Ackermann formula for multivariable control systems", International Journal of Systems Science, pp. 21132127, Jan 31, 2007.

[8] V.V. Pankratov, Y.A. Zima, O.V. Nos, "Selected sections of the automatic control theory", 223 p., 2011

[9] H. Kubota et al., "Regenerating-Mode Low-Speed Operation of Sensorless Induction Motor Drive with Adaptive Observer", IEEE Transaction on Industry Application, Vol. 38, No. 4, pp 1081 - 1086, July/August 2002. 